\title{
Computed tomographic density changes in schizophrenic and manic Nigerian subjects
}

\author{
J.U. Ohaeri, A.O. Adeyinka and B.O. Osuntokun \\ College of Medicine, University of Ibadan, Ibadan, Nigeria \\ Correspondence to: J.U. Ohaeri, Department of Psychiatry, University College Hospital, Ibadan, \\ Oyo State, Nigeria
}

\begin{abstract}
There is paucity of computed tomography (CT) studies from developing countries, where schizophrenia is said to have more favourable outcome and present predominantly with positive symptoms. We highlight the densitometric analysis of frontal and parietal areas, assessed by tracing of regions of interest on the slice that passes through the foramen of Monro and the pineal gland. The relationships of the density values with clinical and CT measures were investigated. Fifty schizophrenics, 14 manic and 41 normal subjects were compared. The schizophrenics were assessed with Andreasen's scale for negative symptoms, and outcome was based on the social and clinical condition in the past year. There were no significant differences in mean ages and brain slice area across the groups. In analysis of variance, schizophrenics and manics had significantly higher frontal density than normals. Schizophrenics had significantly higher parietal density than manics and normals $(p<0.001)$. Parietal density was significantly higher than frontal density, for schizophrenics and normals $(p<0.001)$. For the patients, density values were not significantly correlated with linear CT measures of atrophy (e.g. ventricle: brain area ratio) and duration of illness. Density values were not significantly associated with outcome, presence of negative symptoms, and visual ratings of cortical sulci and central atrophy. Our findings indicate that schizophrenia in developing countries is also associated with non-specific brain abnormalities.
\end{abstract}

Keywords: Computed tomography - Density - Mania - Nigeria - Schizophrenia

\section{INTRODUCTION}

Most studies using computed tomography (CT) with schizophrenics and other psychiatric subjects have focused on measures of ventriculomegaly and cortical sulci atrophy (Pearlsen et al., 1981; Pfefferbaum and Zipursky, 1991). The relatively few reports on densitometric changes have indicated that measures of brain density may be of more differential diagnostic value than measures of cortical sulci and subcortical atrophy (Golden et al., 1981). Examination of brain tissue through measurement of CT density (attenuation) values was used initially in demented patients (Nasser et al., 1980; Albert et al., 1984), in whom reduced parenchymal density accompanied the presence of clinically pathologic lesions. While it was expected that the widely reported ventriculomegaly and cerebral sulci atrophy among schizophrenics would be reflected by hypodensity, studies have produced conflicting results. Some studies reported hypodensity among schizophrenics (Golden et al., 1980, 1981; Kanba et al., 1984), while others reported either

(C) 1995 Rapid Communications of Oxford Ltd no significant differences (Pearlson et al., 1988) or hyperdensity (Dewan et al., 1983; Largen et al., 1984) compared with normal subjects. There are no such reports concerning manic and schizophrenic subjects in developing countries, where schizophrenia has been consistently reported to have a more favourable course and to present predominantly with positive symptoms (Sartorius et al., 1986; Katz et al., 1988; Jablensky et al., 1992). It would be interesting to see whether the differences noted in clinical manifestations of schizophrenia across cultures are also evident in such biological variables as brain CT density.

In this report, we highlight the CT densitometric analysis of frontal and parietal areas of Nigerian schizophrenic and manic subjects attending an acute admission unit, and its relationship with ventricular measures, cortical sulci and central atrophy, age, duration of illness, outcome and presence of negative symptoms.

Behavioural Neurology • Vol 8 . 1995 


\section{METHODS}

\section{Subjects}

The subjects consisted of 50 schizophrenic subjects (62\% male; mean age 35.9 years, S.D. 10.8 years, range $15-61$ years) and 14 manic subjects (43\% males, mean age 34.4 years, S.D. 15 years, range 17-64 years), who were consecutive attenders at the outpatient psychiatric clinic of the University College Hospital, Ibadan (UCH), who fulfilled the study's inclusion criteria. A control group consisted of 41 normal members of the general population $(63.4 \%$ males, mean age 32.61 years, S.D. 11.50 years, range 14-62); these were hospital workers, blood donors, and final year medical students (to match similar social groups among the schizophrenics) who volunteered to have a CT scan in order to help the hospital establish a baseline of values for normal persons.

There were no significant differences in mean ages between each of the experimental (i.e. schizophrenic and manic) groups and the control (ANOVA, $\mathrm{F}=$ $1.7, p=0.17$ ). There were also no significant differences in mean ages between the sexes in each of the groups ( $t$ ranged from 0.9 to $1.2 ; p>0.05$ ), and no significant differences in proportion of sexes across the groups.

The schizophrenic subjects not only fulfilled the Research Diagnostic Criteria for schizophrenia (Spitzer et al., 1975) at their first episodes of illness, but had also attended our facility for at least 1 year, during which time they had at least one relapse of illness in which the symptoms were clearly schizophrenic, without affective or organic features (Ohaeri, 1992). Thus our schizophrenic group did not include subjects with the so-called brief acute psychoses, which are said to be common in the Third World, and which Stevens (1987) had suspected could account for the more favourable course of schizophrenia in developing countries. In other words, subjects chosen were those whose diagnoses had temporal stability (Bellak, 1970). The manic subjects were patients who had attended our unit for at least 1 year, and for whom there was temporal stability in diagnosis. As has been described in our locality, the illness of these subjects had been predominantly characterized by many years of recurrent manic episodes, in which major depressive episodes (DSM-III-R) were hardly evident (Makanjuola, 1985).

All patients were examined during a period of remission, or when they were in a stable condition of chronic psychotic symptoms (not requiring hospitalization or increased medication). Clinical assessments were made by one of us (J.U.O.), using the following instruments: the Brief Psychiatric Rating Scale (Over- all and Gorham, 1962), with symptoms as defined by the glossary of the ninth edition of the Present State Examination (PSE); (Wing et al., 1974); Andreasen's (1982) Scale for Assessment of Negative Symptoms (SANS); the Abnormal Involuntary Movement Scale (AIMS; US Department of Health, 1976a) (to exclude cases with tardive dyskinesia); a modified form of the Mini-Mental State Examination (MMSE; Folstein et al., 1975), which had been used in community studies in our locality (Baiyewu et al., 1993); the scale for assessment of soft signs of neurological dysfunction (PANESS) (US Department of Health, 1976b); and Annett's (1979) handedness questionnaire. These assessments and assignment of outcome category were done in the week before or after CT scan, blind to the results of the scan.

Patients were dichotomized into good and poor outcome groups, based on social and clinical conditions in the past year (Ohaeri, 1993). The most important index was the presence or absence of psychotic symptoms in the past year. As is usual in our country (Jegede, 1981), patients were commonly accompanied to hospital by relations who lived with them, and who willingly volunteered information on the patient's functioning at home. All the patients involved in this study were assessed in the presence of relations living with them. Hence information on the social and clinical functioning of the patients at home could be reliably corroborated.

In view of the consistently noted preponderance of positive symptoms among schizophrenics studied in Nigeria (Sartorius et al., 1986; Katz et al., 1988; Jablensky et al., 1992), and the paucity of negative symptoms among treated cases (McCreadie and Ohaeri, 1993; Ohaeri, 1993), the presence of negative symptoms was operationally defined as the corroboration by relations of the presence of any SANS item at a moderate level of severity, for a continuous 2 month period in the past year. It is to be noted that, even among the patients with negative symptoms, positive symptoms were quite prominent in most cases.

Since we found that all the patients and controls were right handed (as in previous studies; Gureje, 1988), and none of the patients met MMSE criteria for dementia, or had significant severity of soft signs of neurological dysfunction, the subjects were not categorized on these measures. The year of onset of illness and duration of illness were determined as in a previous study (Ohaeri, 1992).

All control subjects were screened for psychiatric disorders by the same assessor, in a PSE-style interview. Subjects chosen were those who had never needed psychological treatment, had no chronic physi- 
cal illnesses (e.g. headache, diabetes melitus or hypertension), and for whom there was no neurological abnormality on detailed physical examination. Also, the psychiatric patient groups had no chronic physical illnesses.

All patients, their relations and control subjects gave consent for the study.

\section{Brain CT measures}

All brain CT scans were performed on a GE 9000 series scanner, by the same group of radiographers, and interpreted by one radiologist who was at all times blind to the clinical status of subjects. Both patients and controls were scanned at each imaging session to eliminate possible systematic bias. Scans showing linear or motion artefact were rejected, and the subjects scanned again. Scanning parameters were kept constant. The scanner receives a 1 day service once a month, by a technician who performs air calibration, phantom centring and calibration according to the CT 9000 manual.

All scans were unenhanced, taken at a $10^{\circ}$ angulation to the canthomeatal line, and with $10 \mathrm{~mm}$ thick slices. The CT measures were mostly in line with the methodology and illustrative pictures kindly provided by the Johns Hopkins Hospital (USA) research group (Pearlson et al., 1989, 1990). All measures were done directly on the image console. The CT measures were obtained by direct tracing of regions of interest and calculation of areas from computerized images on the console.

CT measures involved quantitative assessments and standard CT measures of sulci, central atrophy and ventricular sizes, using illustrative pictures and methods from the USA (Andreasen, 1982; Andreasen et al., 1982a; Pearlson et al., 1989). The following measures were taken from the slice that passed through the foramen of Monro and the pineal gland (P/FOM): the brain slice area (BSA); the bifrontal ratio (BFR); the bicaudate ratio (BCR); frontal horn area: brain ratio (FHA); third ventricle area (TVA); and the densities (attenuation values) of the frontal and parietal lobes.

The slice through the foramen of Monro and the pineal gland, which corresponds to the Schnitzlein and Murtagh (1985) atlas pages 22 and 23, was chosen because it could be easily and reliably identified, contained a minimum of bone artefact, and included cortical and subcortical brain areas believed to be associated with schizophrenia in previous studies. These areas included portions of frontal, parietal and temporal lobes, basal ganglia (including nucleus accumbens), hippocampus, and structures surrounding the third ventricle. The slice is not, however, dominated by ventricle, which might tend to obscure other regional changes in cases of marked dilation (Pearlson et al., 1989).

Lateral ventricle: brain area ratio (VBR) and Sylvian fissure: brain area ratio (SVA) were measured at their widest points, as in previous studies (Andreasen et al., 1982; Pearlson et al., 1989), while visual ratings of cortical sulci (Largen et al., 1984; Pearlson et al., 1989) and central atrophy (Okasha and Madkour, 1982) were also made. The test-retest reliability (intra-rater) for each of the ventricle measures, using 12 scans, was at least $r$ (Pearson's) $=0.9$, while agreements (intra-raters) for the visual ratings ranged from $\kappa=0.67$ to $0.8(p<0.001$; Landis and Koch, 1977).

\section{Density measurements}

We used the methodology of a previous study (Pearlson et al., 1989) to sample areas of interest in the frontal and parietal regions of the brain, at the level of the slice that passes through the foramen of Monro and the pineal gland (P/FOM). At this level, it was easy to avoid bone artefact, ventricle, cerebral sulci, claustrum and caudate nucleus. For each half (i.e. right and left) of the frontal and parietal areas, three samples were taken from regions of interest that contained mixed grey and white matter. By moving a circular region of interest marker to the desired position, the density measures obtained were read directly on the image console. The mean of these measures (i.e. right plus left) was taken as the density for the frontal and parietal lobes, respectively. Reliability (intra-rater) for 12 scans was $r=0.86$ and 0.87 , for the frontal and parietal lobes, respectively.

\section{Data analysis}

Data were analysed to examine: sex differences within each group; differences between psychiatric patient groups; differences between each of the patient groups and controls; and within each group, the relationships between density measures and ventricular measures, visual ratings of cortical sulci and central atrophy, and age, using $t$-tests (two-tailed), analysis of variance, chi-square tests (with Yates's correction where necessary), Pearson's correlation, multiple regression and analysis of covariance (with Turkey's method of multiple comparison) at the $5 \%$ level of significance. In addition, the data of schizophrenics were examined to see differences between outcome groups and the effect of the presence of negative symptoms and duration of illness.

Only the details concerning density measurements and BSA will be presented here, the others having been presented elsewhere (Ohaeri et al., 1995). 
TABLE I. Profile of brain CT densities and brain slice area [mean values (S.D.)]

\begin{tabular}{lccccccc}
\hline & $\begin{array}{c}\text { Normals } \\
(n=41)\end{array}$ & $\begin{array}{c}\text { All schiz. } \\
(n=50)\end{array}$ & $\begin{array}{c}\text { Good } \\
\text { outcome } \\
\text { schiz. } \\
(n=27)\end{array}$ & $\begin{array}{c}\text { Poor } \\
\text { outcome } \\
\text { schiz. } \\
(n=23)\end{array}$ & $\begin{array}{c}\text { No negative } \\
\text { symptoms } \\
\text { schiz. } \\
(n=27)\end{array}$ & $\begin{array}{c}\text { Negative } \\
\text { symptoms } \\
\text { schiz. } \\
(n=23)\end{array}$ & $\begin{array}{c}\text { Manics } \\
(n=14)\end{array}$ \\
\hline $\begin{array}{l}\text { Brain slice } \\
\quad \text { area }\left(\mathrm{mm}^{2}\right)\end{array}$ & $14860(1232)$ & $15027(1419)$ & $14818(1491)$ & $15293(1500)$ & $14670(1345)$ & $15462(1625)$ & $14497(763)$ \\
$\begin{array}{l}\text { Frontal density } \\
\text { Parietal density }\end{array}$ & $25.5(5.13)$ & $31.1(4.6)^{*}$ & $31.8(4.6)$ & $30.5(4.5)$ & $31.5(5.1)$ & $30.5(3.9)$ & $29(4.5)^{*}$ \\
\hline
\end{tabular}

Differences between patients and normal subjects $\left(\mathrm{df}=3 / 101:{ }^{*} F=10.1, p=0.0005 ; * * F=25.8, p=0.0005\right.$.

Differences within groups, between parietal and frontal densities: $\dagger t=4.3, p=0.001 ; \ddagger t=3.7, p=0.001$.

\section{RESULTS}

The mean duration of illness of the schizophrenics (10.9 years, S.D. 7 years, range 1-28 years) was not significantly different from that of manic subjects (9.1 years, S.D. 6 years, range $1-25$ years; $t=0.87$, $\mathrm{df}=64, p>0.05$ ).

Within each of the groups, the only significant sex differences were evident for BSA (Table I), where the mean BSA of males (range of means 15 065-15 726) were significantly higher than those of females (range of means 13 887-14 070) ( $t$ values ranged from 3.1 to 5.2 ; df ranged from 12 to $48, p=0.009$ ). However, across the groups, the BSA values were similar for each of the sexes (i.e. no significant differences between male psychiatric patients and male normal control group, and vice versa), and as already noted, there were no significant differences in proportion of sexes across the groups. Also, analysis of variance (ANOVA) showed no significant differences in BSA across the groups, even with schizophrenics dichotomized into outcome and negative symptom categories $(\mathrm{F}=1.2, p=0.3)$.

Table I shows the profile of BSA, the densities for the groups (including the subgroups of schizophrenics), and the values for tests of significance (ANOVA) between means. The mean frontal densities of all categories of schizophrenics and the manic subjects were significantly higher than that of normal subjects $(\mathrm{F}=10.1, p>0.0005)$, while the higher value of the schizophrenics was not significantly different from that of manic subjects $(t=1.4, p>0.05)$. The mean parietal density of the schizophrenics was significantly higher than that of manic and normal subjects $(\mathrm{F}=25.8, p<0.0005)$, while the higher value of manic subjects was not significantly different from that of normal subjects $(t=1.63, p>0.05)$. Compared with frontal density, the mean parietal density was significantly higher for schizophrenics $(t=4.3$, $\mathrm{df}=98, p<0.001)$, and normal subjects $(t=3.7$, df $=80, p<0.001)$, but was not significantly differ- ent for manic subjects $(t=0.91, p>0.05)$. While the schizophrenics with poor outcome (compared with good outcome), as well as those with negative symptoms (compared with those without negative symptoms), tended to have lower densities, the differences were rather small and insignificant $(p>0.05)$. The mean density values for the manic subjects were consistently in between those obtained for the schizophrenic and normal subjects.

The marked differences in density values is further shown by the fact that $18 \%$ of schizophrenics (versus $2 \%$ and $7 \%$, respectively, of normals and manics) had frontal density values over 2 S.D. of the normal mean. For parietal density, $66 \%$ of schizophrenics (compared with $2 \%$ and $21.4 \%$, respectively, of normals and manics) were over 2 S.D. from the normal mean.

A series of Pearson's correlation and chi-square tests was done, between density values on the one hand, and various CT measures and clinical data on the other hand. Frontal and parietal density values were significantly correlated among all the groups ( $r$ ranged from 0.3 to 0.8 , df $12-48, p<0.05$ ). Although density measures were negatively correlated with brain area, this was significant only in the case of schizophrenics for parietal density $(r=0.29$, $p<0.05$ ). Also, among schizophrenics, age was significantly correlated with frontal density $(r=0.26$, $\mathrm{df}=48, p<0.05$ ), while just failing to reach significance for manic subjects $(r=0.4, p>0.05)$. Among normal subjects, frontal density was significantly correlated with lateral ventricle size $(r=0.3, \mathrm{df}=39$, $p<0.05)$ and Sylvian fissure area $(r=0.8, \mathrm{df}=39$, $p<0.001)$. It is noteworthy that density values were not significantly correlated with duration of illness $(p>0.05)$. Also, density values were not significantly associated with visual ratings of cortical sulci and central (subcortical) atrophy $(p>0.05)$.

In view of these relationships (especially BSA and age), the data were subjected to multivariate statistics. In multiple regression analysis, parietal density was 
TABLE II. Analysis of covariance (ANCOVA) across diagnostic groups

\begin{tabular}{lcccccc}
\hline Covariate & Variable & F ratio & $p$ value & \multicolumn{3}{c}{ Corrected means (S.E.) } \\
\cline { 5 - 7 } & & & & Schiz. & Manics & Normals \\
\hline Brain area & Parietal density & 40.1 & 0.0001 & $35.1(0.5)$ & $30.3(0.9)$ & $28.5(0.5)$ \\
Brain area & Frontal density & 14.7 & 0.0001 & $31.2(0.7)$ & $28.8(1.3)$ & $25.6(0.8)$ \\
Age & Parietal density & 39.1 & 0.0001 & $32.9(0.5)$ & $30.5(0.9)$ & $28.6(0.5)$ \\
Age & Frontal density & 14.9 & 0.0001 & $30.9(0.7)$ & $29.1(1.3)$ & $25.7(0.8)$ \\
\hline
\end{tabular}

The corrected means are with reference to the covariates on the same rows.

significantly predictable by BSA $(t=-2.93$, $p<0.005)$ for schizophrenics; while among manic subjects, parietal density was significantly predictable by bifrontal ratio $(t=2.4, p<0.04)$ and third ventricle area $(t=3.2, p<0.01)$. When the data were subjected to analysis of covariance (ANCOVA) with BSA and age, respectively, as covariates, differences in density values - already highlighted - remained significant (see Table II).

\section{DISCUSSION}

With regards to other CT measures in the larger study, we found that: (1) the larger VBR of psychiatric patient groups just failed to be significantly higher than that of normal subjects; (2) psychiatric patient groups had significantly higher Sylvian fissure area ratio than normal subjects; (3) the third ventricle area of schizophrenics was significantly higher than that of normal subjects; and (4) psychiatric patient groups had significantly higher prevalence of visual ratings of central (subcortical) atrophy (Ohaeri et al., 1995).

Our sample of schizophrenics, while not being representative of the general population, has presented for analysis a sufficient number of subjects with the various clinical features of the disease in our locality. In particular, we were interested in studying the association between disease outcome, presence of negative symptoms and duration of illness on the one hand, and CT measures on the other. While the rather small sample size of manic subjects is a limitation to the inferences that can be drawn from the findings (and probably accounts for the lack of significance in some of the statistical tests), the results have been useful in supporting previous reports, which had shown that CT brain findings of abnormality are not specific to schizophrenia, among the so-called functional psychotic states (Jacoby et al., 1980; Reider et al., 1983). The socio-demographic characteristics of our psychiatric patient groups were similar to those in previous studies in our locality (Makan- juola, 1985; Sartorius et al., 1986; Katz et al., 1988; Ohaeri, 1992, 1993).

While our findings indicated that the hyperdensity reported in some previous studies (Dewan et al., 1983; Largen et al., 1984) is not unique to schizophrenics, it is clear that, whatever might be causing this hyperdensity, it is more severe among schizophrenics than manic subjects. In addition, the abnormality became significantly severe in schizophrenia compared with mania, in the region of interest that borders areas of the brain that have been most implicated in the neuropathology of schizophrenia (Pearlson et al., 1989). The fact that hyperdensity was greater in schizophrenics than manics probably supports Florr-Henry's speculation that the disease process in the psychoses involves a march of pathology from the relatively milder major affective disorders to the more severe schizophrenias (Florr-Henry, 1969).

The methods and area used to measure CT density in schizophrenic patients has varied, so that comparisons between different studies should be made cautiously. Even within this limitation, it can still be said that, when compared with reports from developed countries, our findings show a lot of biological similarities. For instance, our finding of hyperdensity has been widely reported in studies from developed countries (Largen et al., 1983, 1984; Dewan et al., 1983; Rossi et al., 1989). In particular, similar to our findings, the report of Rossi et al., (1989) from Italy indicated that schizophrenics had significantly enlarged third ventricle and hyperdensity in the frontal and parietal regions.

Discussion of the implications of our findings is limited by the fact that no one seems to know what CT density measures mean. Also, there is the problem of beam hardening or spectral shift artefact, a problem to which CT density measurement is prone (Pfefferbaum and Zipursky, 1991). Although this artefact problem was not specifically controlled for, the fact that both patients and controls were scanned at each imaging session should help to eliminate possible 
group bias. Because of this artefact, CT density is influenced by head size, a factor which we corrected for by subjecting the data to ANCOVA.

It has been usual to resort to neuropathological studies to explain hyperdensity among schizophrenics. In histologic sections, Stevens (1982) demonstrated increased fibrillary gliosis that affected principally the periventricular structures of the diencephalon and the periaqueductal region of the mesencephalon or the basal forebrain, in three-fourths of the brain from schizophrenic subjects. Hypothalamus, midbrain tegmentum and substantia innominata were most often affected. She concluded that the nature and distribution of the findings suggest previous or low grade inflammation. In another report, the autopsied brains of schizophrenics and other psychiatric patient groups (mostly with affective features) showed increased callosal thickness (Bigelow et al., 1983). It has been suggested (Largen et al., 1984) that increased density of white matter may be a correlate of thickening of the corpus callosum or of fibrillary gliosis. This hyperdensity has been found to be diffuse in the brain, involving cortical, subcortical and cerebella regions (Dewan et al., 1983), and could reflect a persistent immaturity or altered chemical composition of the neural elements that exist, independent of age-related effects (Dewan et al., 1983). Since frontal density was significantly correlated with age among our schizophrenics, increased mineralization and iron deposits [which Stevens (1982) found in older schizophrenics] might be thought to account for hyperdensity. But in analysis of covariance, differences in density values remained significant, and the relationship of age with density values was not significant for normal subjects. A magnetic resonance imaging study confirmed abnormalities in the anterior right hemisphere white matter, suggesting increased fibrosis or gliosis in this area (Smith et al., 1984). In the study of Dewan et al., (1986), when patients with left ventricle enlargement were compared to those without, a distinct subgroup of schizophrenic patients emerged with mild lateral enlargement associated with increased cortical density, suggesting a pervasive developmental arrest of density decrease during adolescence.

Our finding of no significant correlation between density values and duration of illness is consistent with other reports, indicating that primary structural deficits might already be present in schizophrenics at onset (Golden et al., 1980; Coffman et al., 1984; Kanba et al., 1984; Pearlson et al., 1988). Ultimately, only a neuropathologic study of the brains of Nigerian psychiatric patients can confirm these our findings.
In conclusion, the findings of this study from a developing country indicate that, as in developed countries, schizophrenia and mania are most probably associated with some non-progressive and nonspecific brain pathology.

\section{Acknowledgements}

The University College Hospital, Ibadan, through funds made available to it by the Federal Ministry of Health, as the National Centre of Excellence in Neurosciences, waived the fees for the CT scans. A University of Ibadan Senate Research Grant to one of us (J.U.O.) partially supported the study.

\section{REFERENCES}

Albert M, Naeser MA, Levin HL and Garvey AJ (1984) CT numbers in patients with senile dementia of the Alzheimer's type. Archives of Neurology, 41, 1264-1269.

Andreasen NC (1982) Negative symptoms in schizophrenia: definition and reliability. Archives of General Psychiatry, 39, 784-788.

Andreasen NC, Smith MR, Jacoby CG, Dennert JW and Olsen SA (1982a) Ventricular enlargement in schizophrenia: definition and prevalence. American Journal of Psychiatry, 139, 292-296.

Andreasen NC, Olsen SA, Dennert JW and Smith MR (1982b) Ventricular enlargement in schizophrenia: relationship to positive and negative symptoms. American Journal of Psychiatry, 139, 297-302.

Annett M (1979) A classification of hand preference by association analysis. British Journal of Psychology, 61, 303-321.

Baiyewu O, Bella AF and Jegede RO (1993) The effect of demographic and health variables on a modified form of Mini-Mental State Examination. Journal of Geriatric Psychiatry, 8, 503-510.

Bellak L (1970) The validity and usefulness of the concept of the schizophrenic syndrome. In: The Schizophrenic Reaction (Ed. R. Cancro), p.42. Brunner/Mazel Inc., New York

Bigelow LB, Nasrallah HA and Rauscher FP (1983) Corpus callosum thickness in chronic schizophrenia. British Journal of Psychiatry, 142, 284-287.

Coffman JA, Andreasen NC and Nasrallah HA (1984) Left hemispheric density deficits in chronic schizophrenia. Biological Psychiatry, 19, 1237-1247.

Dewan MJ, Paunduragi AK, Lee SH, Ramachandran T, Levy B, Boucher M, Yozawitz A and Major LF (9(1993) Central brain morphology in chronic schizophrenic patients: a controlled CT study. Biological Psychiatry, 18, 1133-1139.

Dewan MJ, Paunduragi AK, Lee SH, Ramachandran T, Levy B, Boucher M, Yozawitz A and Major LF (1986) A comprehensive study of chronic schizophrenic patients. Acta Psychiatrica Scandinavica, 73, 152-160.

Florr-Henry P (1969) Psychosis and temporal lobe epilepsy: a controlled investigation. Epilepsia, 10, 363-395.

Folstein MF, Folstein SE and McHugh PR (1975) MiniMental State: a practical method of grading the cognitive state of patients for the clinician. Journal of Psychiatric Research, 12, 189-198. 
Golden CJ, Graber B, Coffman J, Berg R, Block S and Brogan D (1980) Brain density deficit in chronic schizophrenia. Psychiatry Research, 3, 179-184.

Golden CJ, Graber B, Coffman JA, Berg RA, Newlin DB and Block S (1981) Structural brain deficits in schizophrenia: identification by CT scan density measurements. Archives of General Psychiatry, 38, 1014-1017.

Gureje O (1988) Sensorimotor laterality in schizophrenia. Acta Psychiatrica Scandinavica, 77, 188-193.

Jablensky M, Korten A, Copper JE and Day Bertelsen A (1992) Schizophrenia: manifestation, incidence and course in different cultures: a WHO ten-country study. Psychological Medicine, Suppl, 20.

Jacoby RJ, Levy R and Bird JM (1980) Computed tomography in the elderly (iii) Affective disorder. British Journal of Psychiatry, 136, 270-275.

Jegede RO (1981) A study of the role of socio-cultural factors in the treatment of mental illness in Nigeria. Social Science and Medicine, 15A, 49-54.

Kanba S, Shima S, Tsukomo D, Masuda Y and Asai M (1984) Brain density in chronic schizophrenia. Biological Psychiatry, 19, 273-274.

Katz M, Marsella A, Dube KC, Olatawura MO, Takahashi R, Nakane Y, Wyne KC, Gift T, Brennan J, Sartorius N and Jabcensky A (1988) On the expression of psychoses in different cultures. Culture, Medicine and Psychiatry, 12, 331-335.

Landis - RJ and Koch GG (1977) The measurement of observer agreement. Biometrics, 33, 59-74.

Largen JW, Calderon M and Smith RC (1983) Asymmetries in the densities of white and grey matter in the brains of schizophrenic patients. American Journal of Psychiatry, 140, 1060-1062.

Largen JW, Smith RC, Calderon M, Baumgarten R, Lu Schoolar JC and Ravichandran GK (1984) Abnormalities of brain structure and density in schizophrenia. Biological Psychiatry, 19, 991-1913.

Makanjuola ROA (1985) Recurrent unipolar manic disorder in the Yoruba Nigerian: further evidence. British Journal of Psychiatry, 147, 434-437.

McCreadie RG and Ohaeri JU (1993) Movement disorders in never and minimally treated Nigerian schizophrenic patients. British Journal of Psychiatry, 164, 184-189.

Naeser MA, Gebhartm C and Levin H (1980) Decreased $\mathrm{CT}$ numbers in patients with presenile dementia. Archives of Neurology, 37, 401-409.

Ohaeri JU (1992) Age at onset in a cohort of schizophrenics in Nigeria. Acta Psychiatrica Scandinavica, 86, 332-334.

Ohaeri JU (1993) Long-term outcome of treated schizophrenia in a Nigerian cohort. Journal of Nervous and Mental Disorders, 18, 514-516.

Ohaeri JU, Adeyinka OA, Enyidah SN and Osuntokun BO (1995) The schizophrenic and manic brains in Nigerian Africans: CT findings. British Journal of Psychiatry, in press.

Okasha A and Madkour O (1982) Cortical and central atrophy in chronic schizophrenia. Acta Psychiatrica Scandinavica, 65, 29-34.

Overall JE and Gorham DR (1962) The Brief Psychiatric Rating Scale. Psychological Reports, 10, 799-812.

Pearlson GD, Veroff AE and Mcttugh PR (1981) The use of computed tomography in psychiatry: recent applica- tions to schizophrenia, manic-depressive illness and dementia syndrome. The John Hopkins Medical Journal, 149, 194-202.

Pearlson GD, Kim WS, Kubos KL, Moberg PJ, Jayaram G, Bascom MJ, Chase GA, Goldfinger AD and Tune LE (1988) Ventricle-brain ratio, C.T. density and brain area in 50 schizophrenics. Archives of General Psychiatry, 46, 690-697.

Pearlson GD, Warren AC, Starkstein SE, Aylward EH, Kumar AJ, Chase GA and Folstein MF (1990) Brain atrophy in 18 patients with Down Syndrome: A C.T. study. American Journal of Neuroradiology, 11, 811-816.

Pfefferbaum A and Zipursky RB (1991) Neuroimaging studies of schizophrenia. Schizophrenia Research, 4, 193208.

Reider RO, Mann LS, Weinberger DR, Vankammen DP and Post RM (1983) CT scans in patients with schizophrenia, schizophrenia affective disorder and bipolar affective disorder. Archives of General Psychiatry, 40, 735-739.

Rossi A, Stratta P, D'Albensio L, Di Michele V, Serio A, Giordano L, Petruzzi C and Casacchia M (1989) Quantitative computed tomographic study in schizophrenia: cerebral density and ventricle measures. Psychological Medicine, 19, 337-342.

Sartorius N, Jablensky A, Korten A, Ernbery G, Anker G, Cooper J and Day R (1986) Early manifestations and first contact incidence of schizophrenia in different cultures. Psychological Medicine, 16, 909-928.

Schnitzelein HN and Murtagh FR (1985) Imaging Anatomy of the Head and Spine: A Photographic Colour Atlas of MRI, CT, Gross and Microscopic Anatomy in Aerial, Coronal and Saggital Planes. Urban and Schwarzenberg, Baltimore, MD.

Smith RC, Baumgartner R, Calderon M, Affas A, Ravichandran GK and Peters I (1985) Magnetic imaging studies of schizophrenia. Psychopharmacology Bulletin, 21, 566-594.

Spitzer RC, Endicott JJ and Robins E (1975) Research Diagnostic Criteria. New York State Psychiatric Institute, New York.

Stevens JR (1982) Neuropathology of schizophrenia. Archives of General Psychiatry, 39, 1131-1139.

Stevens J (1987) Brief psychoses: do they contribute to the good prognosis and equal prevalence of schizophrenia in developing countries? British Journal of Psychiatry, 151, 393-396.

US Department of Health (1976a) Abnormal Involuntary Movement Scale (AIMS). In: ECDEU Assessment Manual for Psychopharmacology (Ed. W Guy), pp. 534537. National Institute of Mental Health, Rockville, MD.

US Department of Health (1976b) Physical and Neurological Examination for soft signs (Paness). In: ECDEU Assessment Manual for Psychopharmacology (Ed. W Guy) pp. 384-406. National Institute of Mental Health, Rockville, MD

Wing JK, Cooper JE and Sartorius N (1974) The Present State Examination, Ninth Revision of the Interview Schedule. Cambridge University Press, Cambridge.

(Received 3 February 1994; accepted as revised 17 January 1995) 


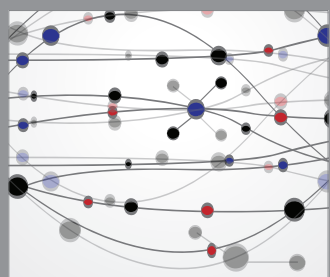

The Scientific World Journal
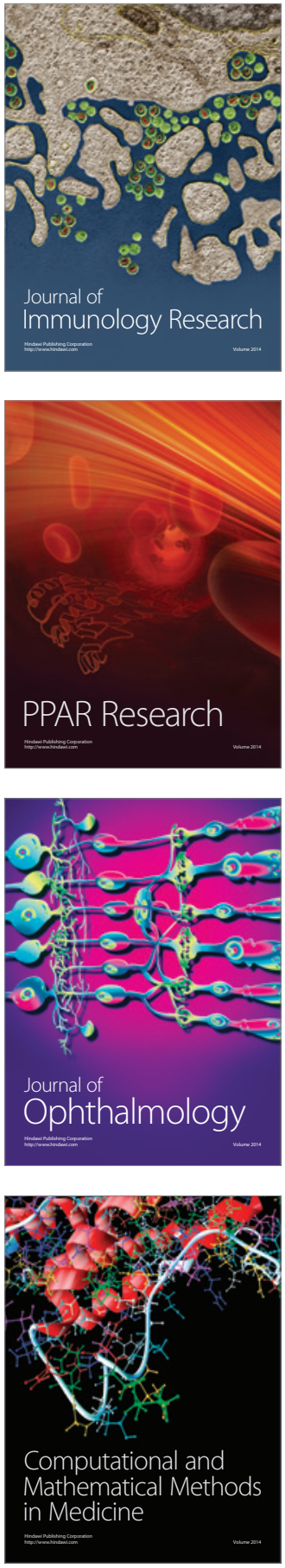

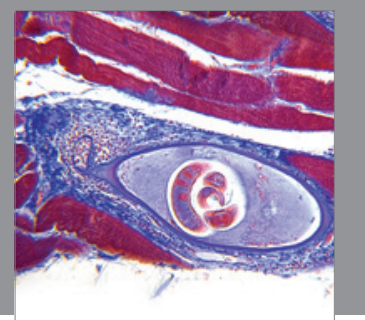

Gastroenterology

Research and Practice
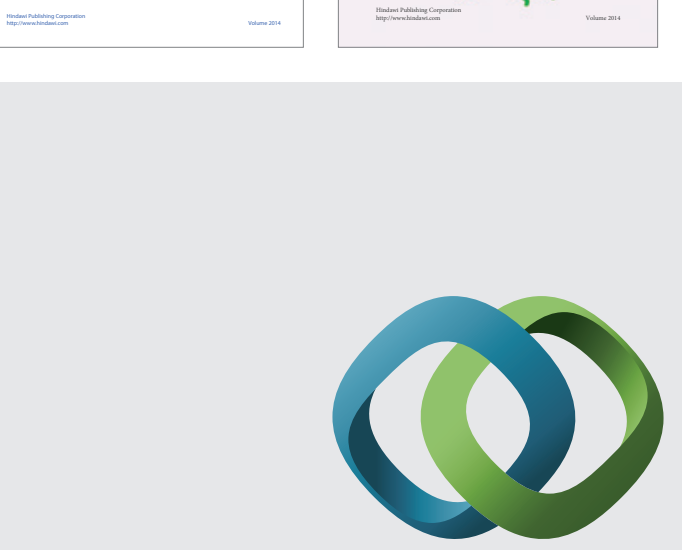

\section{Hindawi}

Submit your manuscripts at

http://www.hindawi.com
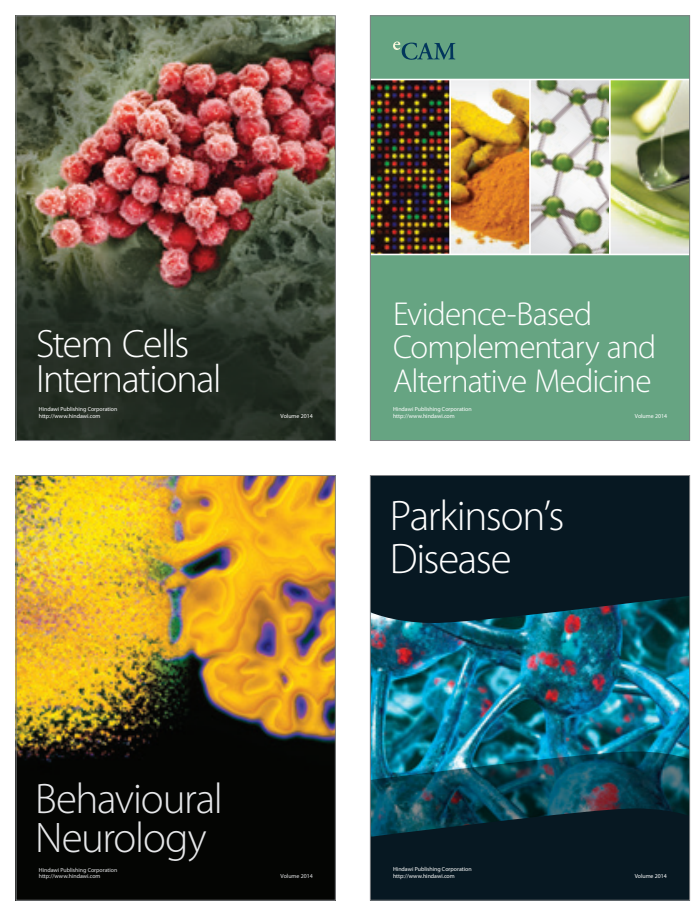

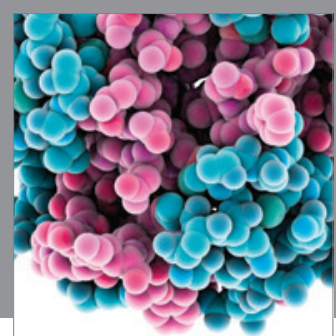

Journal of
Diabetes Research

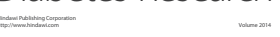

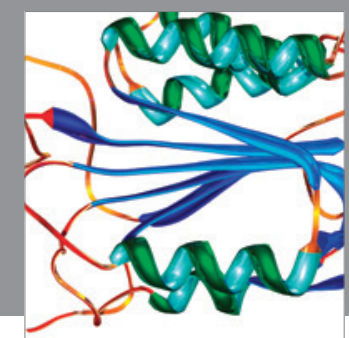

Disease Markers
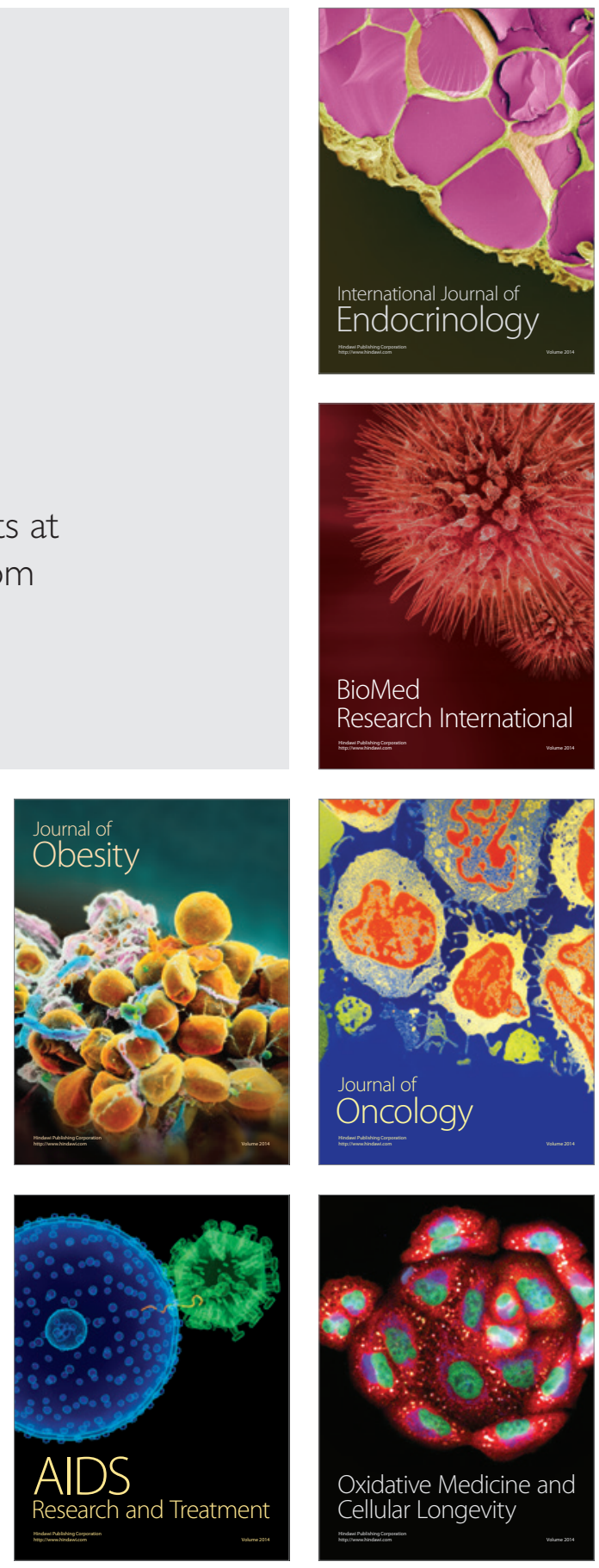